\section{E-050 FIRST-LINE TREATMENT BY EMBOLIZATION WITH INTENTION-TO-CURE IN THE MANAGEMENT OF CRANIAL DURAL ARTERIOVENOUS FISTULAS}

B Cord, S Sommaruga, J Yeung, M Johnson, C Matouk. Neurosurgery, Yale University, New Haven, $C T$

10.1136/neurintsurg-2016-012589.122

Introduction/purpose Cranial dural arteriovenous fistulas (dAVFs) represent abnormal arteriovenous shunts supplied by dural arteries. Their natural history and presentation depend on the pattern of venous drainage encapsulated in the Borden classification. When cortical venous reflux (CVR) is present, aggressive management is mandated. The treatment of cranial dAVFs lacking CVR may be warranted. Treatment modalities include surgical disconnection, radiosurgery, and embolization (alone or in combination). Here we report our early experience in the management of cranial dAVFs using a philosophy of first-line embolization with intention-to-cure.

Materials and methods A series of consecutive cranial dAVFs $(n=14)$ treated by a single operator $(\mathrm{CM})$ at Yale-New Haven Hospital (November 2011 - March 2016) were analyzed. The mean age at presentation was $61 \pm 12$ years $(57 \%$ male). The locations of cranial dAVFs included transverse sinus $(n=6)$, cavernous sinus $(n=2)$, anterior skull base $(n=2)$, straight sinus $(n=2)$, hypoglossal canal $(n=1)$, and midline cerebellar $(n=1)$. Borden types included I $(n=1)$, II $(n=3)$, and III $(n=10)$. Borden types II/III lesions presented most commonly with venous congestion \pm hemorrhage (80\%). A trans-venous approach was prioritized as a first-line treatment strategy. When a trans-venous approach was not possible, as in a majority of type III lesions (80\%), a trans-arterial embolization was performed with intention-to-cure, i.e., penetration of liquid embolic into the draining vein. Mean angiographic and clinical follow-up were $12.4 \pm 12.2$ months (median $=7.1$ months, range $=2.1-40.1$ months ) and $19.4 \pm 15.4$ months $($ median $=13.7$ months, range $=2.1-44.7$ months), respectively. Cure was defined as elimination of CVR on follow-up imaging.

Results The overall cure rate of cranial dAVFs treated using a first-line embolization with intention-to-cure philosophy was 71.4\%. Three patients required an additional treatment modality (2 Gamma Knife radiosurgery and 1 open surgery) resulting in an overall cure rate of $92.6 \%$. A single, elderly patient who presented with a cerebellar hemorrhage died 10 days after a partial trans-arterial embolization due to the severity of her initial hemorrhage. First-line trans-venous embolization resulted in an overall higher embolization-only cure rate (86\%) compared to first-line trans-arterial embolization $(57 \%)$. Both approaches were associated with low permanent morbidity (overall 7.1\%) however the trans-venous approach was fraught with more frequent technical complications, e.g., coil migration. Of note, a single case of trans-venous embolization of a Borden type II transverse sinus dAVF was associated with conversion to a type III lesion complicated by a small, delayed intraparenchymal hemorrhage and new visual field deficit from which the patient completely recovered. Her residual dAVF spontaneously thrombosed resulting in a durable cure.

Conclusions A philosophy of first-line embolization with intention-to-cure of cranial dAVFs is both safe and effective with low morbidity and high, durable cure rates. When possible, a trans-venous route is preferred with higher chances of immediate cure using embolization as a standalone therapy.

Disclosures B. Cord: None. S. Sommaruga: None. J. Yeung: None. M. Johnson: None. C. Matouk: None.

\section{$\mathrm{E}-051$ \\ THE ROLE OF FOLLOW-UP IMAGING AFTER RESECTION OF BRAIN ARTERIOVENOUS MALFORMATIONS IN THE PEDIATRIC PATIENT: A REVIEW}

J Jimenez, Z Gersey, J Wagner, B Snelling, S Ambekar, E Peterson. Neurological Surgery, University of Miami Miller School of Medicine/Jackson Memorial Hospital, Miami, FL

\subsection{6/neurintsurg-2016-012589.123}

Object Pediatric patients are at significant risk for recurrence of brain AVM after resection. While there is general consensus as to the importance of follow-up after surgical resection of an AVM, there is a lack of consistency in follow-up duration. The object of this systematic review is to examine the role of follow-up imaging in detecting AVM recurrence early and preventing AVM rupture.

Methods This systematic review was carried out with articles obtained through a search of the literature according to PRISMA (Preferred Reporting Items for Systematic Reviews and Meta-Analyses) guidelines.

Results Search results revealed 1052 articles, of which 13 studies describing 31 cases of AVM recurrence met criteria for inclusion in this study. Patients receiving follow-up imaging experienced AVM detection significantly earlier (mean $=3.56$ years, $\mathrm{SD}=3.67$ ) than patients with no follow-up $($ mean $=8.86$ years, SD $=5.61 ; \mathrm{p}=0.0085)$. While $13.34 \%$ of patients that were not receiving follow-up imaging presented with rupture of recurrent AVM, 57.14\% of patients that were receiving follow-up imaging presented with ruptured recurrence $(\mathrm{p}=0.019)$.

Conclusions Follow-up imaging has an integral role after AVM resection and is sometimes not carried out for a sufficient period of time, leading to delayed detection of recurrence and increased likelihood of recurrent AVM rupture.

Disclosures J. Jimenez: None. Z. Gersey: None. J. Wagner: None. B. Snelling: None. S. Ambekar: None. E. Peterson: None.

\section{E-052 PERCUTANEOUS SCLEROTHERAPY OF MICROCYSTIC LYMPHATIC MALFORMATIONS - THE USE OF AN INNOVATIVE GRAVITY-DEPENDENT TECHNIQUE}

A Berenstein. Neurosurgery, Mount Sinai Health System, New York, NY

10.1136/neurintsurg-2016-012589.124

Purpose To describe a novel gravity technique of sclerotherapy for microcytic LM.

Methods and technique A 23 angiocatheter connected to a tubing field with the sclerosing agent, is passed through and through the lesion, the angiocatheter is slowly withdraw, with the tubing at 15 $\mathrm{cm}$ of $\mathrm{H} 2 \mathrm{O}$, as a microchannel is entered, the fluid will permeate the microchannel. As the channel(s) fill the tubing needs to be further elevated and that part of the lesion is field, the angiocatheter is slowly further withdrawn, and as a new channel is entered the fluid column starts advancing.

Materials From 2009-2015 in 154 patients with LM, 34 were microcytic, (500 micron range), treated using the gravity technique, with Doxycycline and or Bleomycin

Results Significant improvement was noted in most patients, usually towards the 2 or 3 rd treatment, both clinically and by cross section imaging. No complications occurred.

Conclusion Our gravity technique permits treating lesions with true microcytic channels in a safe and effective manner.

Disclosures A. Berenstein: None. 